\title{
The anatomical variability of the emissary condylar veins, its identification and clinical significance
}

Sir,

We wish to provide some clarification with regard to the paper of Pekcevik et al..$^{[1]}$ about the "Prevalence of clinically important posterior fossa emissary veins on CT angiography" published in your journal. Although the 
computed tomography (CT) angiography is the superior tool to depict the venous structures, especially those with small diameter and the related bony canals, ${ }^{[1]}$ we believe that this minimally invasive technique. Usually fails to represent certain collateral venous channels due to the reverse blood flow and the supine patient position. It is therefore reasonable the fragmentary and description of this paper about the complex normal and variable venous anatomy in the craniocervical area. ${ }^{[2]}$

The identification of the location and course of the emissary condylar veins (CV) (anterior, lateral and posterior) and the anterior condylar confluence inferomedial to the jugular bulb is of immense significance, as they represent the most important connections between the intracranial cerebral and the cervical vertebral venous systems. ${ }^{[3,4]}$ The authors make no mention about the anterior and lateral CV depiction, probably due to the fact that the study performed retrospectively and was not designed to identify all possible venous emissary channels. Further support for our view comes from the following standpoint: "The CV (anterior, lateral and posterior), ${ }^{[3,4]}$ the mastoid and the occipital emissary veins represent the venous connections between the dural venous sinuses of the posterior cranial fossa and the internal jugular vein with the cervical vertebral venous systems". ${ }^{[5]}$

Specifically, the anterior CV appears as a connection of the anterior internal vertebral venous plexus with the internal jugular vein. The anterior $\mathrm{CV}$ usually runs medially through the hypoglossal canal or creates a small anastomosis with the basilar plexus. ${ }^{[6]}$ These emissary veins drains into the lateral part of the marginal sinus that in some cases may bridge the anterior CV with a suboccipital vein. ${ }^{[5]}$ The lateral $\mathrm{CV}$ originating from the medial aspect of the jugular bulb runs posterolateral and is connected with the suboccipital cavernous sinus and the venous plexus around the $\mathrm{V}_{3}$ segment of the vertebral artery (from $\mathrm{C} 2$ vertebra to the dura mater). According to Trolard, ${ }^{[7]}$ its proximal portion may sometimes be found within an osseous canal in front of the occipital condyle. The anterior and lateral CV form a constant structure, the anterior condylar or petrosal confluence ${ }^{[5]}$ that plays a key role in the cerebral blood redirection in the craniocervical area. The posterior CV usually larger from the anterior originates from the superior jugular bulb or occasionally medially from the lower end of the sigmoid sinus. It runs through the posterior condylar canal, drains into the deep cervical vein and also communicates with the horizontal segment of the vertebral artery venous plexus. ${ }^{[1]}$ The lateral and posterior $\mathrm{CV}$ are related to the external vertebral venous system, whereas the anterior $C V$ is related to the internal vertebral venous plexus.

The meticulous knowledge of the very complex venous drainage system of the craniocervical region is necessary especially when treating the dural arteriovenous fistulas of the anterior condylar canal ${ }^{[8]}$ or attempting selective retrograde venous catheterization or interpreting venous imaging studies that show the posterior fossa emissary veins and their variability. Unfortunately, the unrecognized emissary veins of this area may cause diagnostic confusion and surgical complications, which could be simply identified by the direction of the blood flow using Doppler ultrasonography. ${ }^{[6]}$

Konstantinos Natsis, Maria Piagkou

Departments of Anatomy, Medical School, Aristotle University of Thessaloniki, and National and Kapodistrian University of Athens, Athens, Greece

Address for correspondence: Assist. Prof. Maria Piagkou, Department of Anatomy, Medical School, National and Kapodistrian University of Athens, 75 M. Asias street, Athens, 11527, Greece. E-mail: mapian@med.uoa.gr

\section{References}

1. Pekcevik Y, Sahin H, Pekcevik R. Prevalence of clinically important posterior fossa emissary veins on CT angiography. J Neurosci Rural Pract 2014; 5:135-8.

2. Théron J. Cavernous plexus affluents. Neurochirurgie 1972; 18:623-38.

3. Reis CV, Deshmukh V, Zabramski JM, Crusius M, Desmukh P, Spetzler RF, et al. Anatomy of the mastoid emissary vein and venous system of the posterior neck region: Neurosurgical implications. Neurosurgery 2007; 61(Suppl 2):193-201.

4. Jeevan DS, Anlsow P, Jayamohan J. Abnormal venous drainage in syndromic craniosynostosis and the role of CT venography. Childs Nerv Syst 2008; 24:1413-20.

5. San Millán Ruíz D, Gailloud P, Rüfenacht DA, Delavelle J, Henry F, Fasel JH. The craniocervical venous system in relation to cerebral venous drainage. AJNR Am J Neuroradiol 2002; 23:1500-8.

6. Mortazavi MM, Tubbs RS, Riech S, Verma K, Shoja MM, Zurada A, et al. Anatomy and pathology of the cranial emissary veins: A review with surgical implications. Neurosurgery 2012; 70:1312-19.

7. Trolard P. Anatomy of the venous system of the brain and skull. Paris: Thesis of the Faculty of Medicine of Paris; 1868. p. 1-32.

8. Cyril C, Ofélia M, Hervé D. Dural arteriovenous fistula involving the anterior condylar canal. J Neuroimaging 2013; 23:425-8.

\begin{tabular}{|l|l|}
\hline \multicolumn{2}{|c|}{ Access this article online } \\
\hline Quick Response Code: & Website: \\
\hline & www.ruralneuropractice.com \\
\cline { 2 - 3 } & \\
\hline
\end{tabular}

